\title{
A Transition in DNA Molecule's Spatial Ordering Due to Nano-Scale Structural Changes
}

\author{
Yu. M. Yevdokimov ${ }^{\mathrm{a}, *,}$ V. I. Salyanov ${ }^{\mathrm{a}}$, E. V. Shtykova ${ }^{\mathrm{b}}$, K. A. Dembo ${ }^{\mathrm{b}}$, V.V.Volkov ${ }^{\mathrm{b}}$, P.V.Spirin ${ }^{\mathrm{a}}$, \\ A.S.Slusheva ${ }^{a}$ and V.S.Prassolov ${ }^{a}$
}

${ }^{a}$ V.A. Engelhardt Institute of Molecular Biology, Russian Academy of Sciences, ul. Vavilova 32, Moscow, 119991 Russia and ${ }^{b}$ A.V. Shubnikov Institute of Crystallography, Russian Academy of Sciences, Leninski pr. 59, Moscow, 119333 Russia

\begin{abstract}
The optical, X-ray- and AFM-properties of the particles of cholesteric liquid-crystalline dispersion (CLCD) formed by double-stranded DNA molecules and treated with gadolinium salts are studied. It is shown that under certain conditions this treatment results in two effects: i)amplification of the abnormal negative band in the CD spectra specific to the initial CLCD, and ii) disappearance of small-angle X-ray scattering specific of ordered DNA molecules. The explanation of these effects is based on a suggestion that gadolinium ions when present at high concentrations not only induce nano-scale changes in the DNA secondary structure, but also overcompensate the DNA negative charges, influencing the efficiency of DNA-DNA interactions in the content of the particles of CLCD. These processes lead to transformation of the spatial structure in particles of CLCD. The resulting structure of particles of DNA CLCD highly enriched in gadolinium is not "liquid-crystalline"; rather it is a rigid construction of practical importance.
\end{abstract}

Keywords: DNA-based nanostucture, liquid-crystalline dispersions, rare-earth elements, gadolinium ions, rigid structure, circular dichroism.

\section{INTRODUCTION}

The controlled self-assembly of linear nucleic acids (in particular, DNA) or their complexes with biologically or chemically relevant molecules ("guests") is one of the goals of nanotechnology (nanobiotechnolgy) [1]. It is known that linear, double-stranded (ds) DNA molecules can spontaneously condense and to form particles of liquid-crystalline dispersions (LCDs) without precipitating completely out of solution. The existence, shape and size of the formed particles result from a fragile balance between their bulk free energy and their surface free energy. The bulk free energy tends to increase the size of the LCD particles, whereas the surface free energy, which depends strongly on the surface tension between the liquid-crystalline phase and the isotropic phase, tends to reduce the size of the particles. The effect of the DNA charge screening by $\mathrm{Na}^{+}$ions and the increased osmotic pressure induced by neutral polymers such as poly(ethyleneglycol) (PEG) facilitate the formation of the DNA LCD. The competition between the bulk free energy of the particle (which tends to expand the size of the particle) and its surface free energy (which reduces the interface between the DNA rich phase and the surrounding isotropic solution) suggests that there exists a critical size below which the DNA LCD particles are not stable.

*Address correspondence to this author at the V.A. Engelhardt Institute of Molecular Biology, Russian Academy of Sciences, ul. Vavilova 32, Moscow, 119991 Russia; E-mail: yevdokim@eimb.ru
Recently $[2,3]$ we showed that there is a possibility to use the double-stranded (ds) DNA molecules located at regulated distance (about 2.5-5.0 nm) in cholesteric LCDs (CLCDs) particles as the "building blocks" with tailored properties for the formation of nanoconstructions. In contrast to the initial DNA liquid-crystalline particles, the structure of resulting nanoconstruction, consisting of $10^{4} \mathrm{ds}$ DNA molecules, is not "liquid-crystalline" but rather a rigid, crystal-like structure (Fig. 1).

Hence, using the CLCD particles, formed at a fixed osmotic pressure of a solvent, one can realize the transition from the "liquid-crystalline" state of ds DNA molecules to their "solid" state. Since the rigid spatial nanoconstructions (nanostructures) formed by ds DNA molecules possess unique physico-chemical properties [2] and because the dynamic properties of DNA-based nanostructures provide the basis for creation of switchable molecular or supermolecular machines [4,5] (this opens a gate for their wide application in biotechnology and medicine). Our goal was to investigate the "liquid-crystalline" -"solid"- state transition induced by change in the solubility of ds DNA molecules as a result of their interaction with rare-earth cations (RECs).

In this work, detailed investigation of the physicochemical properties of CLCD particles formed by complexes of $\mathrm{ds}$ nucleic acids with RECs was continued. In this study we focuced primerely on the properties of the CLCD in the context of DNA-gadolinium complexes. 

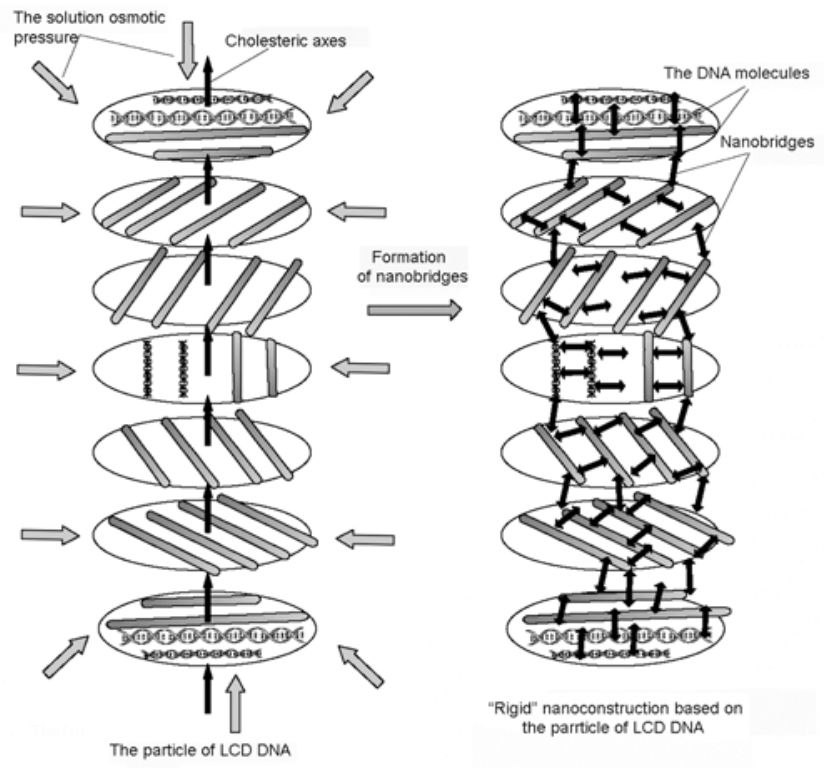

Fig. (1). Principal scheme of transition the DNA-based nanoconstruction from "liquid" to "rigid" state. Double-stranded DNA molecules in neighboring quasi nematic layers in initial liquidcrystalline structure (left) are shown as rods; each layer is twisted in respect to previous one.

\section{MATERIALS AND METHODS}

Ds calf thymus DNA ("Sigma"), which was additionally cleaned of impurities and depolymerized, was used in the experiment. The depolymerization of DNA was performed by an UZDN- 2T ultrasonic disintegrator. The mol. mass of the DNA after ultrasonic depolymerization was equal to (0.6$0.8) \times 10^{6} \mathrm{Da}$, (that corresponds to length, $\mathbf{L}$, of DNA molecules about 300-400 $\mathrm{nm}$ ). We used the rare-earth chloride salts (gadolinium, lanthanum, neodymium, praseodymium, samarium, terbium, and ytterbium; purity of $99.99 \%$ ), which were kindly provided to us from the State Institute of Rare Metals (Moscow, Russia), and poly(ethyleneglycol) ("Fluka," Switzerland, mol. mass 4,000 Da)for these studies.

The formation of the LCDs based on the phase exclusion of ds DNA from PEG-NaCl aqueous solutions was performed as previously described [6]. In the physicochemical sense, the system under investigation is particles of the LCD of the ds DNAs that are isotropically distributed in the aqueous salt solution of PEG. The presence of an abnormal band with a maximum at $\lambda \approx 270 \mathrm{~nm}$ in the circular dichroism (CD) spectrum indicates the formation of particles of the cholesteric LCD (CLCD). The complex of the CLCD with the rare-earth elements (nucleic acid - rare-earth element) was formed by treating the CLCD of the initial ds DNA with aqueous solutions of rare-earth salts.

The absorption spectra of the nucleic acids, the CLCD of the nucleic acids, and the CLCD of complexes (nucleic acid rare-earth element) were taken by a Specord M-40 spectrophotometer (Germany), and the CD spectra were recorded by a portable dichrometer SKD-2 (manufactured by the Institute of Spectroscopy of the RAS, Troitzk, Moscow Region). In all cases, the quartz cells with $1 \mathrm{~cm}$ optical path have been utilized.
The character of ordering of the DNA molecules in particles of CLCD was tested by X-ray diffraction measurements. Taking into account the theoretically estimated, mean diameter of particles of the DNA CLCD (about $0.5 \mu \mathrm{m}$ ) [6] the measuring of X-ray scattering from single LCD particles is practically impossible. To solve this problem, the CLCD particles were concentrated by a well-documented procedure used earlier in the case of CLCD formed by initial DNA molecules or DNA complexed with various biologically active compounds [7-9].

Samples were prepared for X-ray analysis by forming CLCD of initial DNA and CLCD of (DNA-Gd ${ }^{3+}$ ) complexes in large (200-500 ml) volumes, recording their CD spectra, and then concentrating them by low-speed centrifugation (4000 rpm, $30 \mathrm{~min}, 4^{\circ} \mathrm{C}, \mathrm{K} 23$ centrifuge, Germany). Each pellet obtained by centrifugation was placed with a small amount of supernatant in a thin-walled $(0.01 \mathrm{~mm})$ quartz capillary of diameter $0.5 \mathrm{~mm}$ and $\mathrm{X}$-ray diffraction analysis was performed [7-9].

We used an "AMUR-K" small angle X-ray diffractometer manufactured in the Shubnikov Institute of Crystallography of the RAS. Scattering radiation was registered with a linear-position-sensitive proportional detector manufactured in the Institute of Nuclear Physics, Siberian Div. of the RAS, Novosibirsk.

$\mathrm{X}$ - ray small-angle scattering (SAXS) experiments were performed at a wavelength $\lambda=0.1542 \mathrm{~nm}$ in a Kratky-type (infinitely long slit) geometry covered the range of momentum transfer $0.12<\mathrm{s}<7.5 \mathrm{~nm}^{-1}$ (here, $\mathrm{s}=4 \pi \sin \theta / \lambda$, where $2 \theta$ is the scattering angle). The thickness of the sample (about $0.5 \mathrm{~mm}$ ) varied along the length of the sample holder so that the irradiated volume cannot be estimated and thus no absolute calibration was possible. The scattering profiles were corrected for the background scattering and primarily processed using standard procedures.

Comparison of the CD spectra of initial CLCD to the CD spectra of thin films formed by the pellets (phases) obtained from these CLCD and to their X-ray scattering curves was used [6-9] for evaluation of the parameters, reflecting the mode of ordering of the neighboring DNA molecules in phases formed and, hence, in the particles of the CLCD.

The mean repeating distances $\left(\bar{d}=2 \pi / \mathrm{s}_{\max }\right)$ of the periodical motifs (structural elements) in the crystalline regions (that correspond to the peak position $\mathrm{s}_{\max }$ on the scattering patterns) in the scattering curves for the pellets (phases) formed as a result of the procedure above, were calculated using the program PEAK [10].

The meanings of the X-ray parameters used for describing scattering curves, such as the mean long-range order dimension $\boldsymbol{L}$ (the size of crystallites) and the degree of disorder in the system $\Delta / d$ were calculated from the following equations [11]:

$$
\begin{aligned}
& L=\frac{\lambda}{\beta_{s} \cos \theta}, \\
& \Delta / \bar{d}=\frac{1}{\pi} \cdot \sqrt{\frac{\beta_{s} \cdot \bar{d}}{\lambda}},
\end{aligned}
$$


where: $\boldsymbol{\beta}_{\boldsymbol{s}}$ is the full width at a half-maximum intensity of a peak (in radians) observed at a mean scattering angle of $2 \theta$, and $\Delta$ is a mean-square deviation of distances between neighbouring regularly packed structural elements.

The radii of gyration $R_{g}$ and size distribution functions $\mathrm{D}_{\mathrm{V}}(\mathrm{R})$ of structural heterogeneities presented in the DNA CLCD and DNA CLCD treated by gadolinium salt were calculated using an indirect transform program GNOM [12].

Model simulations of two different kinds of structural organizations of the DNA complexes with gadolinium were performed using the program MASSHA. The program is a 3D graphic system to display and manipulate atomic structures and low resolution models. It is coupled with computational modules to allow interactive and automated rigid body refinement against solution scattering data.

The morphology of the ds DNA CLCD particles treated with $\mathrm{GdCl}_{3}$ was examined using a commercial Atomic Force Microscope P47-SPM-MDT (produced by NT-MDT, Russia). To isolate these particles, the solution in which they were formed was filtered through a poly(ethylenetherephtalate) (PETP) nuclear membrane filter with mean size of pores of $0.15 \mu \mathrm{m}$ (produced by the Institute of Crystallography of the RAS), that allows one to immobilize particles; filter was flushed a few times by a distilled water to erase the nonspecifically absorbed $\mathrm{GdCl}_{3}$ and dried in air for no less than $1 \mathrm{~h}[5]$.

\section{RESULTS AND DISCUSSION}

\section{The CD Spectra of Particles of DNA CLCD Treated by Rare-Earth Salts}

As it shown in [13-16], when rare-earth cations interact with linear ds nucleic acid molecules [DNAs or synthetic polyribonucleotide poly(I)- poly $(\mathrm{C})]$, the CD spectra of these molecules change their shape. In particular, when the ionic strength of a solution is low, the CD spectrum characteristic of the linear right-handed B-form DNA with a $\mathbf{r}_{\text {total }}$ of about 0.5 ( $\mathbf{r}_{\text {total }}$ is the ratio of the molar concentration of rareearth cations in the solution to the molar concentration of the DNA nitrogen bases) is similar to the CD spectrum of the left-handed $Z$-form DNA [14,17,18]. When the ionic strength is high (approximately 0.3), this transition is still observed, but its manifestation in the CD spectrum is not so pronounced. Change in the shape of the CD spectrum indicates that the regular character of the secondary structure of linear DNAs is altered, which, according to [13-16], can be a consequence of the formation of complexes of rare-earth cations with pairs of the DNA nitrogen bases and/or with phosphate groups. The induced changes have local character, i.e. they are spreaded over a few base-pairs (about 1-3 nm). Taking into account that sterical limitations are absent in the case of isolated independent DNA molecules, accumulation of many of such changes can completely alter the structural properties of the whole ds DNA molecule $[17,18]$.

Hence, rare-earth cations can easyly induce the local (nano-scale) alteration of ds DNA secondary structure in the case of linear DNA molecules. Such alterations are accompanied not only by a change in the shape of the CD spectrum, but also prevent the formation of the CLCD from somodified ds DNA molecules. This is a typical violation of the regular character of the nucleic acids secondary structure, induced by their complex formation with several metals $[14,15]$.

However, in the case of the CLCD preformed from ds DNA molecules, one can observe quite different effects.

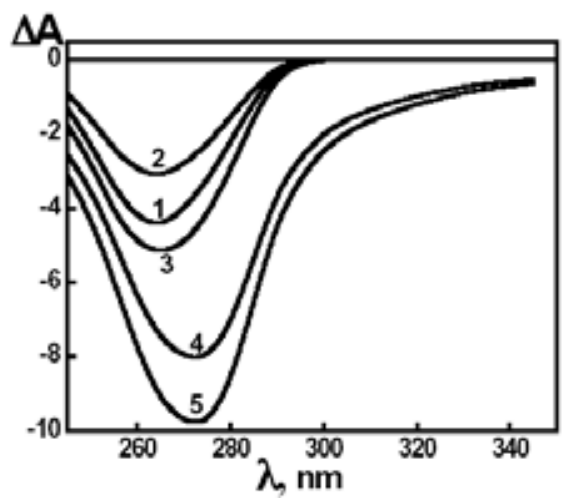

Fig. (2). The CD spectra of the ds DNA CLCD in the absence and presence of $\mathrm{GdCl}_{3}$ in solution.

1 - initial CLCD of ds DNA; $2-0.025 \mathrm{mM} ; 3-0.5 \mathrm{mM} ; 4-1$ $\mathrm{mM} ; 5-2.92 \mathrm{mM} \mathrm{GdCl}_{3}$.

$\mathrm{C}_{\mathrm{DNA}}=10 \mu \mathrm{g} / \mathrm{ml} ; \mathrm{C}_{\mathrm{NaCl}}=0.3 \mathrm{M} ; \mathrm{C}_{\mathrm{PEG}}=170 \mathrm{mg} / \mathrm{ml} ; \Delta \mathrm{A}(\lambda 270 \mathrm{~nm})$ $=A_{L}-A_{R}\left(\right.$ x $10^{-3}$ opt. units $)$.

Fig. (2) compares the CD spectrum of CLCD of the initial DNA (curve 1) formed in water-salt PEG-containing solution to the CD spectra for a CLCD (curves 2-5) treated with $\mathrm{GdCl}_{3}$. One can remind that every particle of CLCD contains about $10^{4}$ ds DNA molecules fixed on distances within 2,5-5,0 $\mathrm{nm}$ (depending on osmotic pressure of the solvent [6]

The CD spectrum of the DNA CLCD differs in shape from the spectra of all sets of secondary structures characteristic of this macromolecule. The formation of DNA LCD is clearly accompanied by the appearance of an intense band in the $\mathrm{CD}$ spectrum in the region where the DNA nitrogen bases absorb. The band in the CD spectrum for DNA CLCD has a shape identical to that of the DNA absorption, but the maximum of the band in the CD spectrum is "red" shifted ( $\lambda$ $\sim 270 \mathrm{~nm}$ ).

The theoretical and experimental data [19-21] were used to identify the factors determining the sign and amplitude of the intense band in the CD spectrum of the DNA CLCDs. These factors were summarized in [8]. Here one can remind only the most important:

1) The amplitude of the intense band is directly proportional to the concentration of DNA molecules used for formation of dispersion. Normalized with respect to the DNA concentration and expressed as $\Delta \varepsilon_{270}$, the amplitude of this band (in otherwise identical conditions) is constant and is about 150 units. This value which characterizes the optical activity of the DNA nitrogen bases in the structure of LCD is far greater than the value of $\Delta \varepsilon(\sim 2.5$ units $)$ characteristic of the molecular optical activity of the nitrogen bases in the structure of isolated linear DNA molecules, calculated theoretically and measured experimentally [19-21] 
2) The amplitude of the intense band in the CD spectrum depends on the size (diameter) of particles of LCD and increases with its growth,

3) The amplitude of the intense band depends on the helical pitch of the cholesteric structure. The smaller the pitch of the helical structure, i.e. the greater the angle between neighboring "quasi-nematic" layers (Fig. 1) of DNA molecules in the cholesteric structure, the greater the amplitude of the band in the CD spectrum and visa versa.

4) The sign of the intense band in the CD spectrum of DNA CLCD in the absorption region of its chromophores (nitrogen bases) depends on the angle of their inclination in respect to the long axis of the DNA molecule.

5) The amplitude of the band in the CD spectrum depends on the anisotropy of the DNA molecule and negligible changes in the properties of the DNA secondary structure are accompanied by diminishing of the amplitude of intense band in the CD spectrum.

Hence, the formation of ordered, spatially twisted structure of neighboring DNA molecules in particles of CLCDs as a result of phase separation in polymer-containing solutions is accompanied by the appearance of specific, intense band in the CD spectrum in the region of the DNA nitrogen bases absorption. Taking points " $1-5$ " above into account and in order to stress the difference between molecular and "structural" circular dichoism, the term an "abnormal band" was used to signify this band in the CD spectrum [19].

According to theoretical calculations, the appearance of this band unequivocally testifies the macroscopic cholesteric twist of neighboring DNA molecules in particles of LCD. The negative sign of the band in the CD spectrum proves the left-handed cholesteric twist of the right-handed DNA molecules (B-form) in these particles. The intense band in the CD spectrum (Fig. 2, curve 1) located in the absorption region of the nitrogen bases of the DNA molecule, in our case negative, is the direct evidence for the formation of the CLCD characterized by helically twisted spatial structure $[22,23]$, or a so-called cholesteric structure [24] of particles of LCD and the term CLCD (cholesteric liquid-crystalline dispersion) was used to signify these particles.

It is worth noting that particles of the DNA CLCD exist at certain osmotic pressure of the aqueous salt solution specified by a high PEG concentration in the solution [9].

Fig. (2) shows as well that the amplitude of an abnormal negative band $(\lambda \sim 270 \mathrm{~nm})$ in the CD spectrum characteristic of the initial ds DNA CLCD is changed and its maximum is displaced to the red area of the spectrum as a result of treatment of this CLCD with $\mathrm{GdCl}_{3}$.

It should be noted that complexes between the rare earth ions and the phosphate groups have a low solubility $[13,16]$ and the particles of the ds DNA CLCD treated by these ions begin slowly to precipitate. This process is accompanied by decrease in the amplitude of the abnormal band. However, the intensive shaking of the solution containing the obtained pellet results in a full restoration of the initial abnormal band in the CD spectra of the CLCDs, i.e. the coalescence of the particles of these CLCDs does not happen.

The gadolinium-concentration dependence of the abnormal band amplitude $\left(\boldsymbol{\Delta A}_{\mathbf{2 7 0}}\right)$ in the CD spectrum of the DNA CLCD particles is shown in Fig. (3). One can see that the amplitude of the abnormal band in the CD spectrum of particles of the CLCD is only slightly changed at low concentrations of $\mathrm{GdCl}_{3}\left(\mathbf{r}_{\text {total }}<0.5\right)$. Under these conditions the noticeable alterations in the CD spectra of the linear DNA treated by gadolinium salts are observed [13-18]. The violation of regular character of the secondary structure of linear molecules nucleic acids which is accompanied by change in the shape of their CD spectra is specific not only for to their complex formation with several metals $[14,15]$ but complexes with gadolinium [5 ].

Fig. (3) unequivocally means that low concentrations of gadolinium ions can induce the alteration of the secondary structure of DNA molecules even fixed in the content of particles of CLCD.

One can see as well that a quite sharp change in the amplitude of the abnormal band in the CD spectrum of the CLCD of the DNA-Gd ${ }^{3+}$ occurs when there is large excess of gadolinium cations in the solution $\left(\mathbf{r}_{\text {total }}>20\right)$ and its maximum is shifted by $10 \mathrm{~nm}$ toward long wavelengths.

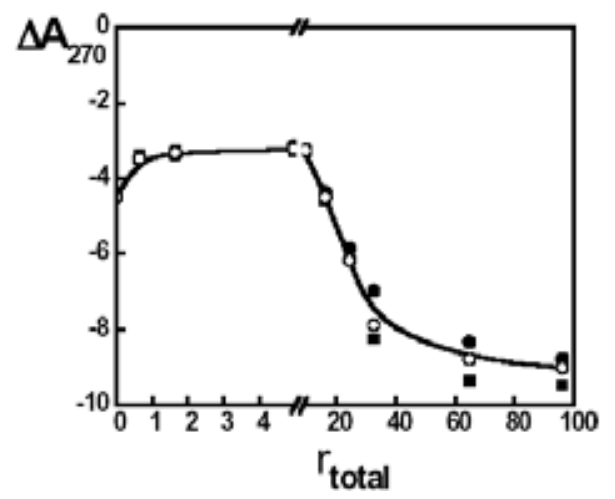

Fig. (3). The dependence of the amplitude of the abnormal negative band in the CD spectra of the CLCD formed by (DNA-Gd ${ }^{3+}$ ) complexes upon $\mathrm{r}_{\text {total }}$ value.

$\mathrm{C}_{\mathrm{DNA}}=10 \mu \mathrm{g} / \mathrm{ml} ; \mathrm{C}_{\mathrm{NaCl}}=0.3 \mathrm{M} ; \mathrm{C}_{\mathrm{PEG}}=170 \mathrm{mg} / \mathrm{ml}$; $\Delta \mathrm{A}(\lambda 270$ $\mathrm{nm})=\mathrm{A}_{\mathrm{L}}-\mathrm{A}_{\mathrm{R}}$ ( $\times 10^{-3}$ opt. units).

$\mathrm{r}_{\text {total }}$ - is the ratio of total $\mathrm{GdCl}_{3}$ molar concentration to the molar concentration of the DNA bases.

The increase in the amplitudes of abnormal bands in the CD spectra occurs within a very short time, more exactly, simultaneously with the CLCD treatment by the rare earth salts. Similar alterations in the shape of the CD spectra of the CLCD of the DNA are observed when these CLCDs are treated with high concentrations of salts of other rare-earth element (lanthanum, neodymium, samarium, and terbium).

The observed increase in the amplitude of the negative band and the change in the shape of the CD spectrum of the DNA CLCD are similar to changes in the CD spectra of this CLCD upon cross-linking of neighboring DNA molecules due to the formation of nanobridges between them [25]. The formation of such nanobridges leads to the disappearance of the "liquid" character in the packing of DNA molecules in 
the particles of the CLCD and results in the formation of the rigid spatial structure [2].

Comparison of Fig. (2) to Fig. (3) allows one to suppose, that the change in the shape of the CD spectrum can be considered as evidence that the interaction of rare-earth ions with DNA molecules fixed in the spatial structure of particles of the CLCD, has a complex character. It is not excluded, that after local changes in the DNA secondary structure induced at a low extent of $\mathrm{Gd}^{3+}$-cation interactions with the DNA, an additional process closely related with the mutual orientation of neighboring DNA molecules in the particles of the CLCD takes place.

It is worth noting that the treatment of particles of the DNA CLCD with the salts of other three-valence cations (iron, aluminum, etc.) is accompanied by the sharp decrease in the amplitude of the abnormal band in the CD spectrum rather than by the amplification of this band.

Therefore, for rare-earth cations, there is a specific mechanism responsible for the amplification of abnormal band in the CD spectrum of CLCD. The results above speak in favor of the assumption that the amplification of the abnormal bands in the CD spectra is connected to the reasons, which differ from reasons [13-16] responsible for the alteration of the shape of the CD spectra of linear ds DNA, treated with gadolinium salts.

In the framework of the theory [19] that describes the abnormal optical properties of the particles of the initial CLCD formed by ds DNA molecules, one can expect that there are two main reasons responsible for amplification of the abnormal band in the CD spectra.

Taking into account that separation of chains of the ds DNA molecules in the content of particles of the CLCD is impossible due to the sterical reasons [26,27], the first reason is the increase in the diameter of the initial CLCD particles induced by interaction with gadolinium ions. The second reason is the change of the pitch of the cholesteric structure of the ds DNA CLCD as a result of interaction of gadolinium ions with these molecules

Hence, it is of interest, first, to evaluate the possible shape of particles of the CLCD DNA-gadolinium complexes. It is known that the interaction of $\mathrm{Gd}^{3+}$ ions with polyphosphates results in a poorly soluble compound [16]. Applied to the phosphate groups of the ds DNA molecules, this basically means that at high $\mathrm{Gd}^{3+}$ concentrations the CLCD particles have reduced their solubility.

\section{Visualization of Particles of the CLCD of the DNA-Gd Complex}

Particles of the CLCD of the initial ds DNA do not exist in the absence of high osmotic pressure of the solvent (induced by high PEG concentration. Therefore their fixation on a nuclear membrane filter and visualization is impossible under these conditions. However, if poorly soluble CLCD particles consisting of molecules of the DNA-Gd complex are formed then the immobilization of these particles on the surface of the nuclear membrane filter becomes possible and the size and shape of these particles can be investigated.
Fig. (4) shows the AFM image of ds DNA CLCD particles after their treatment with $\mathrm{GdCl}_{3}$ and immobilization on nuclear membrane filter (PETP). One can see that these particles exist as independent, individual objects that can be easily visualized. The existence of independent particles implies the appearance of an uncompensated positive surface charge on particles of the CLCD that prevents their aggregation.

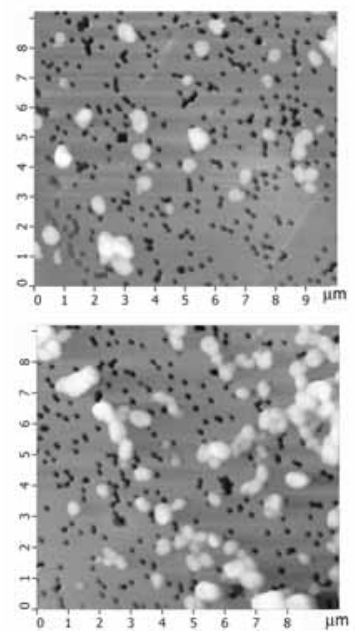

Fig. (4). AFM images of the particles of CLCD formed by (DNA $\mathrm{Gd}^{+3}$ ) complexes and immobilized onto the surface of the nuclear membrane filter (PETP).

$\mathrm{C}_{\mathrm{DNA}}=1.07 \mu \mathrm{g} / \mathrm{ml} ; \mathrm{C}_{\mathrm{NaCl}}=0.03 \mathrm{M} ; \mathrm{C}_{\mathrm{PEG}}=17 \mathrm{mg} / \mathrm{ml} ; \mathrm{C}_{\mathrm{GdCl}}=0.23$ $\mathrm{mM}$.

(The dark spots are "pores" in the nuclear membrane filter. Two sites on the PETP filter are shown.).

Fig. (5) demonstrates the size distribution of these particles as well as the pores in the filter. One can see that the mean size of particles makes $(4.5-5.0) \times 10^{2} \mathrm{~nm}$, i.e. the mean diameter of the ds DNA CLCD particles after treatment with gadolinium is comparable with the mean diameter of initial ds DNA CLCD particles [6,28].

Hence, the results of AFM measurements allow one to suppose that the neutralization of phosphate groups of $\mathrm{ds}$ DNA molecules fixed in the content of CLCD by the rare earth ions is not accompanied by alteration in the mean size of the CLCD particles. This results is important because it suggests that the average packing density of the DNA molecules in particles of the CLCD of the DNA-Gd complex is similar to the packing density of the DNA molecules in particles of the CLCD formed from the initial molecules. These results further imply that the mean concentration of chromophores (DNA nitrogen bases) retained in particles of the CLCD of the DNA-Gd complex is high, and is sufficient for holding the high optical activity of particles of [19] and the first reason (page (4)) to explain the amplification of the abnormal band in their CD spectra must be rejected.

The visualization of single particles indicates, that when particles of the CLCD of the ds DNA are treated with the $\mathrm{GdCl}_{3}$ solution, the "liquid" character of DNA molecules packing in these particles disappears and particles acquire a rigid spatial structure. Such rigid structure can reflect not only a decrease in the solubility of DNA molecules, but as 
well that there is strong attractive interaction between the fragments of neighboring DNA molecules.

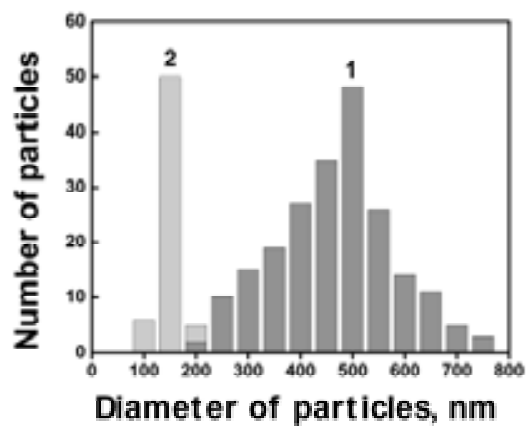

Fig. (5). Size distribution of the ds DNA CLCD particles treated by $\mathrm{GdCl}_{3}(1)$ and the pores (2) in the membrane filter.

$\mathrm{C}_{\mathrm{DNA}}=1.07 \mu \mathrm{g} / \mathrm{ml} ; \mathrm{C}_{\mathrm{NaCl}}=0.03 \mathrm{M} ; \mathrm{C}_{\mathrm{PEG}}=17 \mathrm{mg} / \mathrm{ml} ; \mathrm{C}_{\mathrm{GdCl} 3}=0.23$ $\mathrm{mM}$.

One can summarize the results shown above as the following. The treatment of the ds DNA CLCD with $\mathrm{GdCl}_{3}$ is accompanied by a neutralization of phosphate groups in the DNA molecules by gadolinium cations. Disappearance of the fluidity of the ds DNA CLCD particles results in a shortrange attractive interaction between the fragments of the DNA molecules. The existence of independent particles speaks in favor of an appearance of noncompensated positive surface charge on the CLCD particles. This, in turn, prohibits the coalescence of these particles and the creation of a uniform macroscopic phase, i.e. is accompanied by the stabilization of spatial structure of individual particles of the CLCD.

\section{X-Ray Parameters of Phases of the CLCD of the DNA- Gd Complexes}

The rigid, low-solubility characteristics of particles of CLCD of the DNA-Gd complexes provides the opportunity concentrate them by low-speed sedimentation and therefore the study of their X-ray parameters.

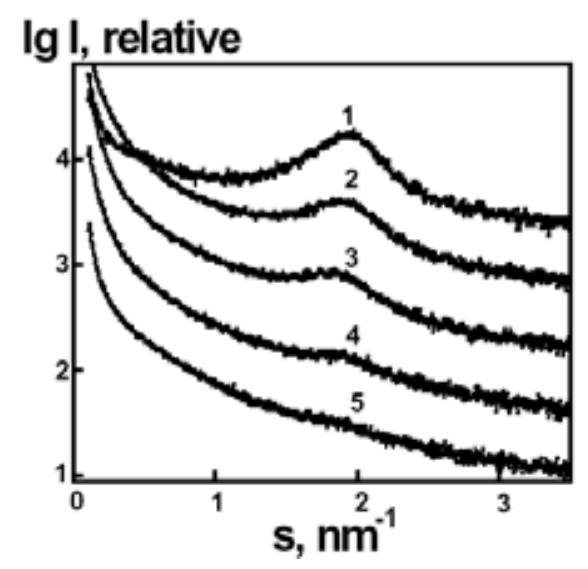

Fig. (6). The small-angle $X$ - ray scattering curves of the phases prepared as a result of low-speed sedimentation of the CLCD particles formed by initial DNA and by (DNA-Gd ${ }^{+3}$ ) complexes.

$1-\mathrm{r}_{\text {total }}=0 ; 2-\mathrm{r}_{\text {total }}=0,082 ; 3-\mathrm{r}_{\text {total }}=0,167 ; 4-\mathrm{r}_{\text {total }}=0,33 ; 5-\mathrm{r}_{\text {total }}$ $=0,66$;

$\mathrm{C}_{\mathrm{NaCl}}=0,3 \mathrm{M} ; \mathrm{C}_{\mathrm{PEG}}=170 \mathrm{mg} / \mathrm{ml}$.
Fig. (6) compares the X-ray scattering curve for the pellet (phase) prepared from initial DNA CLCD to curves for phases formed by CLCD (DNA-Gd ${ }^{3+}$ ) complexes. The scattering curves (1) for the pellet (phase) formed by procedure described above (page 3) and having an abnormal optical activity of negative sign (Fig. 2) show only one distinct Bragg reflection. The absence of highest orders of the small angle reflection in the X-ray scattering patterns points to the absence of a regular three dimensional order in the arrangement of the DNA molecules, i.e. the phase has no ideal crystalline structure. This means that only "short-range" orientational order, typical of liquid-crystalline phases prepared from DNA molecules, can exist in the arrangement of neighboring DNA molecules [29-31].

Fig. (6) shows as well that experimental curves for phases formed by CLCD (DNA-Gd ${ }^{3+}$ ) complexes are characterized by Bragg peaks at $\mathrm{s}=1.9 \mathrm{~nm}^{-1}$, and by a scattering at very small angles (central scattering). The presence of central scattering points to the formation of nano-scale structural heterogeneities in the samples, while thecharacteristic Bragg peaks correspond to a quasi-crystalline order in the specimens under study. It can be easily seen that an increase in the gadolinium concentration in the samples leads to the disappearance of the diffraction maximum on the X-ray scattering curve. This effect has not been previously shown for the CLCD of nucleic acids. A similar disappearance of the maximum on scattering curves is observed for the CLCD of complexes of DNA with other rare earth elements (lanthanum, neodymium, holmium, and ytterbium). In Fig. (6) one can see that the amplitude of the Bragg reflection maximum (characteristic of spatial ordering) become nearly zero at $\mathbf{r}_{\text {total }}>0.5$.

Size distribution functions of structural heterogeneities presented in the DNA specimen and in the complexes of DNA with gadolinium calculated by the program GNOM show that all samples demonstrate bimodal distributions with main fraction consisting of scattering particles characterized by radii of about $5-6 \mathrm{~nm}$ and wide maximum with dimensions from about 10 up to $50 \mathrm{~nm}$. Most probably, that the first fraction of obtained particle size distributions corresponds to a cross-section of molecular ensemble of DNA, and the second one to their size. This size corresponds to the size of particles of the CLCD evaluated by AFM (Fig. 4).

The characteristics of the ordered regions in these systems were computed from the Bragg peaks in the scattering patterns by fitting Gaussian profiles to interactively selected peaks using the program PEAK. The obtained data are shown in Table $\mathbf{1}$. Table $\mathbf{1}$ presents the X-ray parameters of the phases formed from the DNA-Gd ${ }^{3+}$ complexes for various $\mathbf{r}_{\text {total }}$ values. As one can see from Table 1, amplitudes of the Bragg peaks gradually decrease along with the increasing gadolinium concentrations in the CLCD. These results show a clear loss of the quasi-crystallinity of these samples..

Comparison of Fig. (6), Fig. (3) to Table 1 clearly shows that even small amounts of $\mathrm{Gd}^{3+}$ bound to DNA molecules in CLCD are capable of destroying the translational order in the arrangement of adjacent molecules. However this process is accompanied only by negligible changes in the abnormal optical activity of CLCD (Fig. 3). At much higher amounts of bound $\mathrm{Gd}^{3+}$ (Fig. 3) takes place quite other process, re- 
Table 1. Structural Characteristics of the Initial DNA CLCD And CLCD Treated by Gadolinium

\begin{tabular}{|c|c|c|c|c|c|c|c|}
\hline Samples & $\mathbf{S}_{\mathbf{m a x}}, \mathbf{n m}^{-1}$ & $\mathbf{d}, \mathbf{n m}$ & $\mathbf{L}, \mathbf{n m}$ & $\mathbf{\Delta} / \mathbf{d}$ & $\mathbf{A}$ & $\mathbf{S}$ & $\mathbf{r}_{\text {total }}$ \\
\hline \hline 1. & 1,9 & 3,2 & 21 & 0,12 & 0,52 & 0,0127 & 0 \\
\hline 2. & 1,9 & 3,3 & 28 & 0,11 & 0,30 & 0,0057 & 0,082 \\
\hline 3. & 1,9 & 3,3 & 30 & 0,10 & 0,22 & 0,0026 & 0,167 \\
\hline 4. & 1,9 & 3,3 & 26 & 0,10 & 0,05 & 0,0005 & 0,33 \\
\hline 5. & 1,9 & 3,3 & 24 & 0,10 & 0,02 & 0,000 & 0,66 \\
\hline
\end{tabular}

A - amplitude of the Bragg peac, relative units.

$\mathbf{S}$ - area under Bragg peac, relative units.

sponsible for increase in an abnormal optical activity, despite disappearance of small-angle X-ray peaks (Fig. 6).

The mean height of the Bragg peak on the X-ray scattering curves of the phases of the DNA - gadolinium complexes as a function of $\mathbf{r}_{\text {total }}$ was analyzed. The analysis shows that, when the $\mathrm{Gd}^{3+}$ concentration is equal to $0.5-0.6 \mathrm{Gd}$ atoms per DNA base, the spatial order of the neighboring DNA molecules, which is characterized by the Bragg-peak height (or the area under the scattering curve) disappears, in contrast to particles of the initial CLCD formed from intact molecules of ds DNA characterized by the 3.1-3.2-nm Bragg reflection $[6,31]$ corresponding to the mean distance between the double stranded DNA molecules (B-form) ordered in particles of the CLCD. Nevertheless, the abnormal band in the CD spectrum of the CLCD of the DNA-Gd complexes holds, indicating the cholesteric order of chromophores of DNA molecules bonded in a complex with gadolinium. Comparison of Fig. (3) to Table 1 shows as well that the amplification of the abnormal negative band in the CD spectrum of the CLCD of the DNA-Gd complexes takes place at high gadolinium concentrations in the solution $\left(\mathbf{r}_{\text {total }}>5\right)$, i.e., this happens under conditions of full absence of the translational order of the DNA molecules in particles of the CLCD.

\section{Putative Explanation for the Amplification of the Ab- normal Band in the CD Spectra of the CLCD Complexes of DNA With Rare-Earth Elements}

The above results show that multivalent Gd-ions are not simply agents to screen the negative charges of the DNA phosphate groups. In the case of the CLCD these ions play a complex role in influencing DNA-DNA interactions.

Two peculiarities characteristic of particles of the CLCD of the DNA-Gd complex, as well as complexes of the DNAs with other rare-earth element (lanthanum, neodymium, holmium, and ytterbium), differ these particles from particles of the CLCD formed by complexes of the DNA with cations of other metals. First, it is the disappearance of the maximum on the X-ray scattering curves in samples of the CLCD from complexes of nucleic acids with rare-earth elements. Second, it is amplification of the abnormal band in the CD spectra of CLCD that occurs at saturating concentrations of rare-earth cations bound to the DNA.

There are two principal reasons to explain the disapperance of X-ray maxima in the scattering curves.
First, noncompensated positive surface charge on the ds DNA molecules arising as a result of interaction multivalent $\mathrm{Gd}^{3+}$ with ds DNA molecules in the content of particles of CLCD can result in disappearance of their ability to coalescence. Under these conditions, the formation of "united" phase in the course of low-speed centrifugation of particles of CLCD formed from (DNA- $\mathrm{Gd}^{3+}$ ) complexes is not possible. Because the value of positive surface charge depends on gradual rise in concentration of $\mathrm{Gd}^{3+}$ ions, this effect can result in gradual decrease of the X-ray maxima in the scattering curves. However, data presented in Fig. (3) and Table 1 clearly demonstrate that disappearance of the X-ray maxima takes place at very low concentrations of $\mathrm{Gd}^{3+}$ ions, which are not capable of providing noncompensated positive surface charges for all ds DNA molecules in the content of CLCD. Hence, this reason seems to be impossible.

Second, the interaction multivalent $\mathrm{Gd}^{3+}$ with $\mathrm{ds}$ DNA molecules in the content of CLCD result in nano-scale modification of parameters of the secondary structure DNA molecules. This process is accompanied by destruction of parallel orientation of densely packed DNA molecules, and, hence, by disappearance of the X-ray maxima in the scattering curves. To analyze the second reason more carefully, it is necessary to take into account the following: as it was shown previously [13-18], when the rare earth cations are bound to linear ds DNA molecules, the shape of the CD spectrum of these molecules changes sharply at $\mathbf{r}_{\text {bound }} \approx 0.5$. This change demonstrate the loss of the regular homogeneous character typical of the secondary structure of DNA. Such alteration can be associated with the local (nano-scale) conformational transition, for instance, of B-Z type. The change in dependence of a so-called the "CD-melting temperature" $\left(\boldsymbol{\tau}_{\text {melt }}\right)$ of the CLCD upon increase of the gadolinium concentration [17] speaks in favor of a conformational change in the DNA molecules despite the packing of the DNA molecules in particles of the CLCD. These facts argue that the binding of gadolinium ions to ds DNA molecules even packed in particles of the CLCD at low concentrations leads to a nano-scale alteration in homogeneity of the secondary structure of the DNA. "Modified" nucleic acid molecules are separated into alternating fragments differing in conformations (e.g., B-ZB-B-Z- Z- etc. for DNA). The junctions between B-DNA and Z-DNA fragments also contain extruded bases [32], providing the sites with modified nano-size properties. The existence of modified DNA with the B-form fragments is confirmed by the fact that planar antibiotics (daunorubicin, mitoxantrone) can be incorporated between the base pairs of the 
modified DNA in the particles of the CLCD of DNA complexes with gadolinium.

If the fragments of the neighboring molecules of the complexes of the DNA with gadolinium or even all of the molecules packed in particles of the CLCD acquire heterogeneous secondary structure, the translational order of these fragments (molecules) is broken resulting in disappearance of the small-angle reflection on X-ray patterns as is observed in our experiment (see Fig. 6). Indeed, general characteristics of the peaks such as peak position $\mathbf{s}_{\max }$ on the scattering patterns, repeating distances of the periodical motifs in the crystalline regions $\bar{d}=2 \pi / \mathrm{s}_{\max }$, mean long-range order dimension $\boldsymbol{L}$ (the size of crystallites) and the degree of disorder in the system $\Delta / \bar{d}$ do not vary; demonstrating that the structure of parallel packed DNA molecules is constant. However, consecutive reduction of the areas under Bragg peaks after the addition of gadolinium to the DNA CLCD reflects a decrease in the number of ordered quasi-crystalline regions in the whole system. At the same time, we observe the central scattering intensification, which reflects the formation of a new type of nano-scale structural heterogeneity, which are most likely conglomerates of DNA-Gd complexes without parallel ordering. Thus, X-ray data show that the treatment of the DNA CLCD by gadolinium destroys the typical parallel orientation in the dense packing of DNA molecules and leads to a formation of disordered regions.

To confirm that parallel orientation of the densely packed DNA molecules is changed, model simulations of different kinds of structural organizations of the LCD of DNA complexes with Gd under these conditions were performed. Two possible structural changes affecting the scattering patterns were considered: a disorder of parallel packaging, and a bending of the DNA rods in the position of Gd location in the DNA-Gd complexes. Theoretical calculations in the framework of various models allow one to conclude:

1) Increasing in the degree of disorder in the packaging of the DNA rods leads to a decrease in the amplitude of the maximum on the corresponding model curve. Further disordering results in a decrease the Bragg peak on the scattering curve and even to full disappearance of its maximum.

2) A bending of the DNA molecules as a result of Gd binding is accompanied by the same effect: increasing of the number of bends causes a decrease in the amplitude of the Bragg peak and the complete disappearance of the maximum.

These experimental results confirm the assumption that the treatment of particles of the CLCD with rare-earth cations leads to the appearance of the modified secondary structure of the DNA molecules.

Under saturating gadolinium concentrations, the negative charges of the phosphate groups of DNA are neutralized by positively charged gadolinium cations. According to data obtained by various physical methods $[17,33]$, other process also occur simultaneously.

First, as a consequence of the gadolinium ions neutralizing effect the negative charges of the phosphate groups in the DNAs CLCD became insoluble. Indeed, in the presence of large excess of gadolinium ions, these ions displace the so- dium ions, are bound to the phosphate groups of the DNA. It is worth noting, that when $\mathrm{Gd}^{3+}$ ions are bound to polyphosphates, that is poorly soluble Gd-polyphosphate is formed (solubility constant is equal to about $10^{-12} \mathrm{M}$ ) [16,34]. Since ds DNA molecules have a "polyphosphate" origin, these molecules in the presence of saturating gadolinium concentrations become poorly soluble in PEG-salt-aqueous solutions. Under these conditions, the stable spatial structure of dispersion particles is possibly formed and the presence of PEG is not required to stabilize the structure of particles of the CLCD. Moreover, gadolinium ions, neutralizing the charges of the phosphate groups of the DNA create an excess positive surface charge on particles of the CLCD and aggregation of these particles becomes impossible. This behavior is corroborated by the atomic force microscopic data according to which these gadolinium-ion-treated particles of the CLCD are independent objects (see Fig. 4). Indeed, the appearance of a noncompensated positive charge on the ds DNA molecules will induce an attraction between the neighboring DNA molecules. The overall surface charge on the CLCD particles further results in the stabilization of the spatial structure of these particles after their treatment with $\mathrm{GdCl}_{3}$. This means that, as a result of interaction of gadolinium ions with the ds DNA in the content of the CLCD particles, these particles lose their ability to coalescence. Further more under these conditions particles of the CLCD lose their solubility and sediment in the solution. The sedimentation is accompanied by a decrease in the amplitude of the abnormal band in the CD spectrum. However, the intense shaking of the solution containing the resulting sediment leads to the complete recovery of the initial abnormal optical activity; i.e., the aggregation of neighboring particles of the CLCD of the DNA-Gd complex does not occur. The tenfold dilution of the CLCD formed in the PEG-containing solution $\left(\mathrm{C}_{\mathrm{PEG}}=\right.$ $170 \mathrm{mg} / \mathrm{ml}$ ) of the DNA-Gd complex does not lead to a change in the abnormal optical properties of the dispersion (with allowance for the decrease in the concentration of particles of the CLCD upon such dilution).

Thus, particles of the CLCD of the DNA whose phosphate groups are neutralized by gadolinium ions become poorly soluble and can exist in the absence of a high osmotic pressure inthe PEG-containing solution. This means that the osmotic pressure of the aqueous salt (or aqueous) solution is sufficient for supporting the cholesteric mode packing of molecules of the DNA-gadolinium complexes in particles of the CLCD. Such particles can be immobilized on a membrane filter, and their shape can be probed after washing with water to prevent $\mathrm{NaCl}$ crystallization and/or PEG film formation. Fig. (4) displays the AFM images of the filterimmobilized DNA-Gd CLCD particles. One can see that the mean size of these particles practically coincides with that for particles of native DNA CLCD. The latter fact is quite important, because it means that the mean DNA packing density is not changed by $\mathrm{Gd}$ incorporation, i.e., the chromophore density in such dispersions remains high enough to sustain their abnormal optical activity.

The intriguing questions are why the amplitude of the anomalous CD band does not change at low occupancy $(\mathbf{r}<$ 1) in the DNA-Gd CLCD and why it increases a high occupancy $(\mathbf{r}>\mathbf{2 0})$. 
Here one can remind the following. It is well-known that molecular motions in classical low-molecular compounds are free, and hence, the corresponding X-ray scattering peaks are almost absent. The theories describing an appearance of abnormal optical activity of low-molecular cholesterics $[22,35,36]$ assume that the sufficient conditions for abnormal optical activity are the "orientation" ordering of chromophores of molecules in quasinematic layers of molecules and their helical ordering. The theory usually do not connect the mode of crystallographic packing of neighboring lowmolecular compounds and the orientation order of their chromophores in the liquid-crystalline structure [37]. But, in either case an appearance of abnormal optical activity requires dense chromophore packing.

By contrast, according to the presented above data, the $\mathrm{X}$-ray peaks indicate that the molecular motions in the initial CLCD formed by DNA molecules are significantly frozen. Hence, in the case of DNA molecules, the relation between the nitrogen base (chromophores) orientation about the long axis of DNA molecules in each quasinematic layer and the orientation order of the bases in the CLCD particle can be quite complex. Assuming the theoretical requirements above and taking into acoount data on the same packing density in CLCDs of native and Gd-containing DNA, we can suppose that small amounts of Gd ions can locally disturb the DNA secondary structure (Fig. 3) but, inasmuch as the chromophore packing density and orientation order in the cholesteric are nor altered, in general, the abnormal optical activity of the dispersions under these conditions can be changed, but only nonsignificantly (Fig. 7).

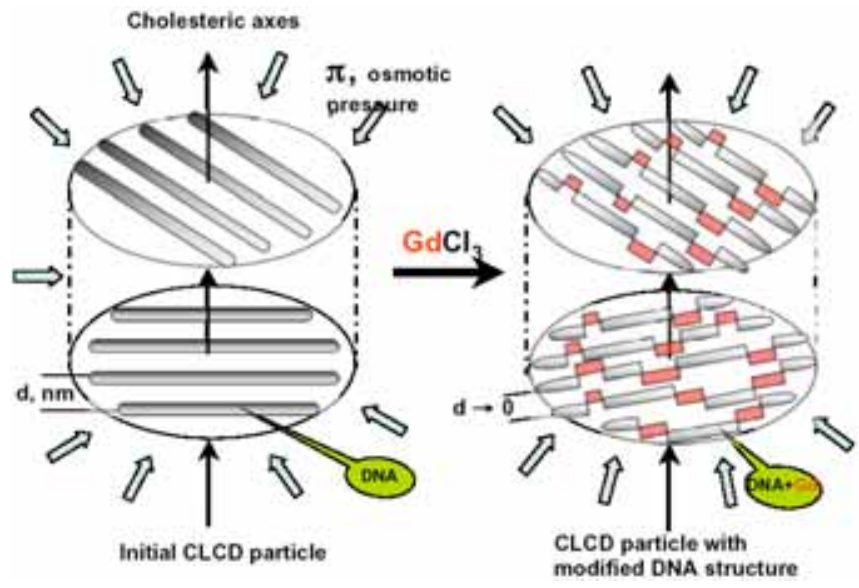

Fig. (7). Scheme of minor alterations in the structure of the particle of the CLCD as a result of treatment of the ds DNA molecules with a low concentration of gadolinium salt. Note, that under these conditions the secondary structure of the ds DNA begins to modify, the mean distance between neighboring DNA molecules begins to change as a result of the interaction of gadolinium ions with DNA, however the twist angle of neighboring quasi nematic layers is not altered. Osmotic pressure is still needed for stabilization of this structure.

Second, nonuniform distribution of gadolinium ions over the surface of DNA molecules results in a heterogeneous secondary structure, that is an irregular attraction between the fragments of the neighboring DNA molecules, fixed initially at about $3.3 \mathrm{~nm}$ (Table 1). The physical origin of this attraction mediated by a strong correlation interaction is related to either electrostatic or entropic contributions. In addition, such interactions are, probably, influenced due to the interlocking $\mathrm{Gd}^{3+}$ ions, referred to "counterion cross-links". Here one should $\mathrm{n}$ stress that the intermolecular interactions are quite different in the DNA molecules with different counterions and conformations [38].

Under conditions of high Gd-ion concentration situation is changed dramatically.

Gd-ions, when present at high concentration, are capable of overcompensating the DNA charges, and the DNA charge inversion can take place, inducing a change in the spatial structure of the CLCD. Indeed, the separation of chains of the ds DNA molecules in the content of particles of the CLCD is impossible due to the sterical reasons [26,27]. The synergic effect of the nano-scale alterations of the secondary structure and the charge inversion of the DNA molecule change the mode of spatial packing of these molecules in the particles of CLCD (Fig. 8). Under conditions of dense packing, the electrostatic forces between DNA molecules, vhich are intimately connected with helical nature of charge distribution, are large enough to overcome intrinsic forces that stabilize the initial cholesteric structure specific to B-form structure [39-41]. This can alter the helical twisting of the cholesteric structure, i.e., facilitate the change of the cholesteric pitch, $\mathbf{P}$.

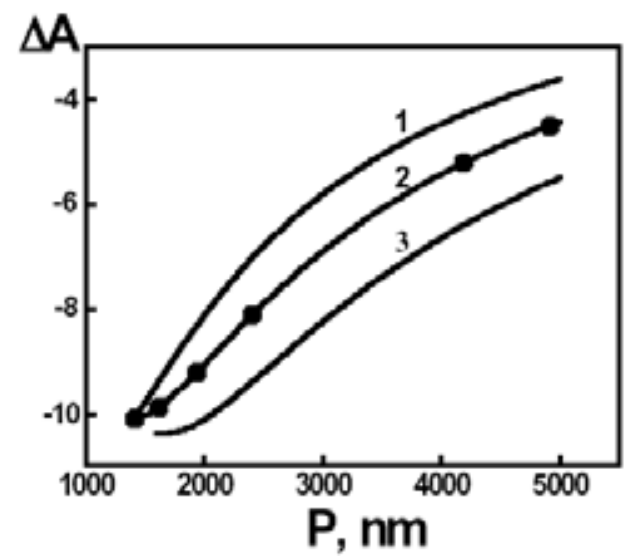

Fig. (8). The theoretical dependences (continuous curves) of the maximal amplitude, $\Delta \mathrm{A}$, of the band in the $\mathrm{CD}$ spectra of the DNA CLCD treated with $\mathrm{GdCl}_{3}$, upon the pitch, $\mathrm{P}$, value.

The black points correspond to the experimentally measured $\Delta \mathrm{A}$ values.

$\lambda=270 \mathrm{~nm} ; \mathrm{C}_{\mathrm{DNA}}=10 \mu \mathrm{g} / \mathrm{ml} ; \mathrm{C}_{\mathrm{NaCl}}=0.3 \mathrm{M} ; \mathrm{C}_{\mathrm{PEG}}=170 \mathrm{mg} / \mathrm{ml}$;

Curve $1-\mathrm{D}=350 \mathrm{~nm}$, curve $2-\mathrm{D}=450 \mathrm{~nm}$, curve $3-\mathrm{D}=550 \mathrm{~nm}$;

$\Delta \mathrm{A}(\lambda 270 \mathrm{~nm})=\mathrm{A}_{\mathrm{L}}-\mathrm{A}_{\mathrm{R}}$ ( x 10-3 opt. units).

To confirm this statement, the theoretical evaluations based on the theory [19] were performed. This theory permits to describe the optical properties of particles of ds DNA CLCD treated with $\mathrm{GdCl}_{3}$, in particular, the correlation between an abnormal optical activity of the ds DNA CLCD and $\mathbf{P}$ value of the cholesteric structure formed by DNA molecules in the content of these CLCD. Assuming that in the case of the ds DNA CLCD the diameter of particles, D, is 
about $0.4 \mu \mathrm{m}$, the initial $\mathbf{P}$ value is about $3 \mu \mathrm{m}$ and the initial $\Delta \mathbf{A}$ value is about $4000 \times 10^{-6}$ optical units, the theoretical curve for $(\boldsymbol{\Delta} \mathbf{A}-\mathbf{P})$ relationship was calculated at the beginning (Fig. 8). This curve clearly shows that the decrease in $\mathbf{P}$ value is accompanied by the increase in $\mathbf{\Delta} \mathbf{A}$ value, i.e. the smaller the pitch of a cholesteric structure, i.e. the greater the twist, the more intense is the amplitude of an abnormal band in the CD spectrum. The experimental meanings of $\Delta \mathbf{A}$, i.e. the observed amplitudes of the abnormal band in the CD spectra of the CLCD formed by ds DNA and treated with $\mathrm{GdCl}_{3}$, correspond fairly well to the theoretical curve (Fig. 8). Comparison of the experimental results and the theoretical calculation confirms the supposition about correlation between the decrease in $\mathbf{P}$ value and the increase of an abnormal optical activity of the CLCD particles treated by the gadolinium salt.

Then, taking into account the known concentration of gadolinium, a correlation between the $\mathbf{P}$ values and gadolinium concentration in used solutions was estimated (Fig. 9). Hence, the "P-effect", i.e. an amplification of the abnormal band in the CD spectra as a result of the alteration of the helical twist of the cholesteric structure of the ds DNA CLCD, is existing in the system under study.

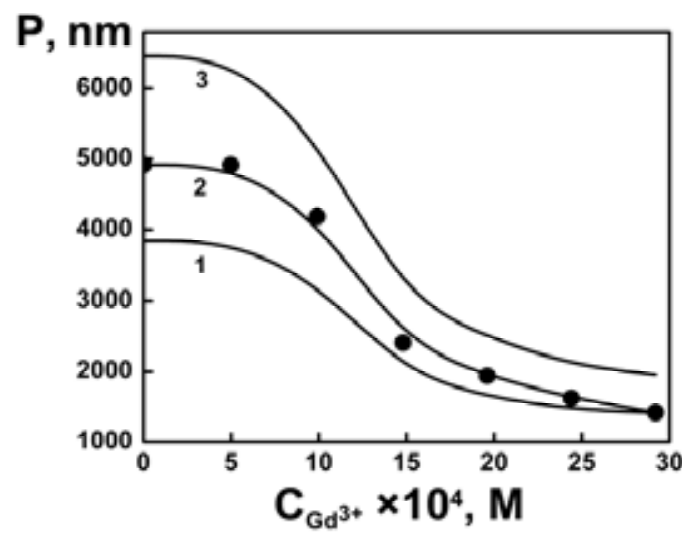

Fig. (9). The theoretical dependences of the $\mathrm{P}$ value of the cholesteric structures for the ds DNA CLCD particles upon the $\mathrm{GdCl}_{3}$ concentration.

$\mathrm{C}_{\mathrm{DNA}}=10 \mu \mathrm{g} / \mathrm{ml} ; \mathrm{C}_{\mathrm{NaCl}}=0.3 \mathrm{M} ; \mathrm{C}_{\mathrm{PEG}}=170 \mathrm{mg} / \mathrm{ml}$;

Curve $1-\mathrm{D}=350 \mathrm{~nm}$, curve $2-\mathrm{D}=450 \mathrm{~nm}$, curve $3-\mathrm{D}=550 \mathrm{~nm}$.

One can see, that here the spatial ordering of neighboring DNA molecules in quasi-nematic layers is practically absent; besides, under these conditions the twist angle between neighboring DNA quasi-nematic layers is increased (the Pvalue is decreased, right structure). However, under these conditions, strong interaction between the fragments of neighboring DNA molecules can result not only in stabilization, but in decrease of solubility of whole structure. The "rigid" structure appears and it can exists in absence of an osmotic pressure of the solvent.

Hence, the change in the angle of twist between neighboring DNA molecules fixed in the structure of.

CLCD is supported by results of the theoretical calculations according to which an increase in the twist angle (decrease in $\mathbf{P}$ value) of the cholesteric helical structure of the initial DNA molecules is accompanied (Fig. 10), by an amplification of the amplitude of the abnormal negative band in the $\mathrm{CD}$ spectrum. At high extent of Gd ion binding to the DNA, not only the negative charges of phosphate groups are neutralized by the Gd cations, but the altered surface charge distribution makes an additional contribution to the chiral interaction between adjacent DNA-Gd molecules in the dispersion particles [40-42].
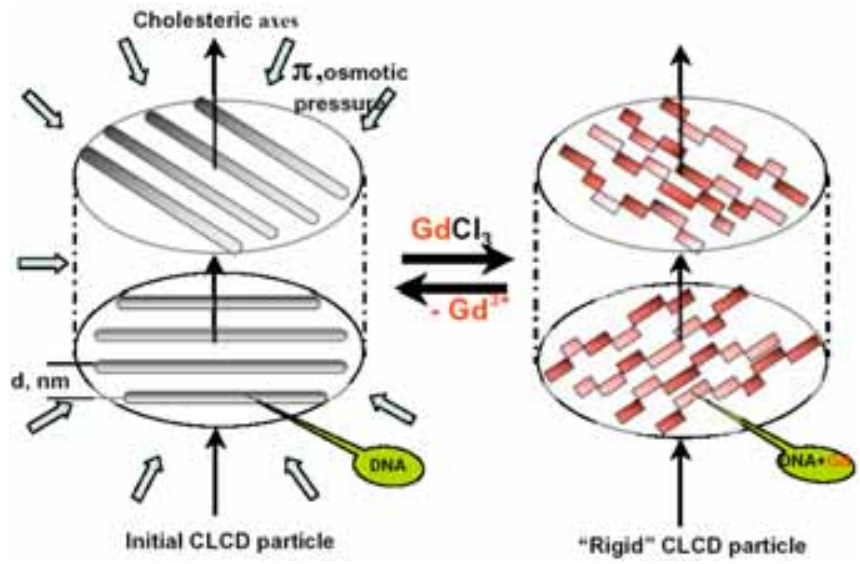

Fig (10). Scheme of transition from "liquid" to "rigid" structure of the particle of the CLCD induced by a high $\mathrm{Gd}^{3+}$ concentration. One can see, that here the spatial ordering of neighboring DNA molecules in quasi-nematic layers is practically absent; besides, under these conditions the twist angle between neighboring DNA quasi nematic layers is increased (the P-value is decreased, right structure). However, under these conditions, strong interaction between the fragments of neighboring DNA molecules can result not only in stabilization, but in decrease of solubility of whole structure. The "rigid" structure appears and it can exists in absence of an osmotic pressure of the solvent.

It is important to emphasize that the problem of the correlation between the local heterogeneous conformation of the structure of nucleic acid molecules and the character of their packing in dense packed liquid-crystalline structures remains a subject of active theoretical investigations [40,43-46]. Change in the twisting of the cholesteric structure can occur in the form of a phase transition. It is worth noting that the possibility of the phase transition between two cholesteric forms was recently analyzed by V. Golo et al. [47]. However, such a transition in our case can be quite diffuse (Fig. 3) due to the smallness of the system elements.

Thus, the combination of two different effects determines the unique optical properties of the CLCD of the complexes of the DNA with gadolinium. The first effect is the formation of nano-scale heterogeneities in the secondary structure of the DNA molecules interacting with gadolinium. The second effect is the enhancement of the interaction between the modified DNA molecules. The first effect is responsible for the "nematization" of the properties of the DNA molecules in quasinematic layers in the structure of the particles of CLCD formed by DNA-gadolinium complexes, i.e. for the disappearance of the small-angle X-ray scattering. The second effect is responsible for the large twisting of the cholesteric spatial structure of the CLCD particles, i.e. the amplification of the negative band in the CD spectrum. Similar unique properties are characteristic of the CLCD particles of the DNA treated with other rare-earth cations (lanthanum, neodymium, holmium, and ytterbium). 
The data obtained in this work show that rare-earth cations interacting with ds nucleic acid molecules fixed in the structure of particles of the CLCD induce nano-scale alterations in the secondary structure of nucleic acids. The accumulation of these alterations is accompanied by a change in the DNA-DNA interactions results in a spontaneous change in the helical twisting of the cholesteric structure of the CLCD. This means that the "cholesteric-1" (Fig. 10), determined by the combination of properties of the initial nucleic acid molecules (in particular, the conformation of the nucleic acid, charge of phosphate groups and the mode of charge distribution in the DNA helical grooves, etc.) is spontaneously transformed to more strongly twisted "cholesteric2" (Fig. 10) depending on a new set of properties for the "modified" nucleic acid. Therefore, the transition between two spatial structures of particles of the CLCD occurs. The observed transition is reversible, since the addition of ethylenediaminetetraacetic acid (EDTA) which binds the gadolinium ions transforms the "cholesteric-2" to the initial "cholesteric-1". Change in the DNA cholesteric pitch implies a reorganization of the whole spatial structure of the particles of the CLCD. Therefore, a "DNA machine", i.e. particle of the CLCD, consisting of $10^{4}$ spatially ordered nucleic acid molecules, makes work. This means that there is a possibility for transformation of the energy of the interaction between gadolinium ions and ds nucleic acid molecules into the work of the microscopic machine consisting of nucleic acid molecules fixed in the spatial structure of particles of the CLCD, which is easily detected by the change in the abnormal optical activity.

Finally, interaction of ds DNA with $\mathrm{Gd}^{3+}$ ions in the CLCD particles yields a stable biomaterial which is highly enriched in gadolinium and the concentration of which can be assessed by the measurements of the abnormal negative band in the CD spectrum. The existence of independent CLCD particles enriched with rare earth cations opens the possibility of a wide range medical and biotechnological application of these particles.

\section{CONCLUSION}

Thus, the data obtained in this work show that the rareearth cations interacting with the ds nucleic acid molecules induce two different structural effects. First, for linear DNA molecules, this interaction is accompanied by change in the parameters of the secondary structure of the nucleic acid molecules realized within nano-scale length. It is not excluded that the observed nano-scale structural transition for the B-form DNA is similar to the known B-Z transition. Second, for the DNA molecules fixed in the structure the CLCD of particles, the accumulation of these structural alterations coinsides with the reduced solubility of the DNA molecules. These conditions induce not only strong attraction between neighboring DNA molecules but also the spontaneous change in the helical twisting of these molecules. This means that in this case, the alterations in the ds DNA structure induced by treatment with gadolinium are "transformed" into a change in the mode of spatial packing of the neighboring DNA molecules. This process results in the amplification of the abnormal band in the CD spectrum, as well as changes in other physical parameters of the CLCD particles. The fine details of this process are now under study.

\section{REFERENCES}

[1] Seeman, N.C. Annu. Rev. Biophys. Biomol. Struct., 1998, 27, 225.

[2] Yevdokimov, Yu. M.; Sitchev, V.V. Open Nanosci. Rev., 2007, 1, 1.

[3] Yevdokimov, Yu. M.; Sitchev, V.V. Rus. Chem. Rev., 2008, 77.

[4] Mao, C.; Sun, W.; Shen, Z.; Seeman, N.C. Nature, 1999, 397, 144.

[5] Yevdokimov, Yu. M.; Salyanov, V. I.; Kondrashina, O. V.; Gasanov, A. A.; Shtykova, E. V.; Dembo, K. A. J. Exptl. Theor. Phys., 2007, 104, 499.

[6] Yevdokimov, Yu. M.; Skuridin, S. G.; Lortkipanidze, G.B. Liq. Cryst., 1992, 12, 1.

[7] Yevdokimov, Yu. M.; Skuridin, S.G.; Salyanov, V.I. Liq. Cryst., 1988, 3, 1443 .

[8] Yevdokimov, Yu. M.; Salyanov, V.I.; Dembo, A.T.; Spener, F. Sensory Systems, 1999, 12, 151.

[9] Yevdokimov, Yu. M.; Salyanov, V.I. Liq. Cryst., 2003, 30, 1057.

[10] Konarev, P. V.; Volkov, V. V.; Sokolova, A. V.; Koch, M. H. J.; Svergun, D. I. J. Appl. Crystallogr., 2003, 36, 1277.

[11] Vainshtein, B.K. Diffraction of X-rays by Chain Molecules, Elsevier Publ. Comp., Amsterdam-London-N-Y., 1966.

[12] Svergun, D. I. J. Appl. Crystallogr., 1992, 25, 495.

[13] Haertle, T.; Augustyniak, J.; Guschbauer, W. Nucleic Acids Res., 1981, 9, 6191.

[14] Gruenwedel, D. W.; Cruikshank, M. K. J. Inorg. Biochem., 1991, 43, 29.

[15] Rosetto, F. E.; Nieboer, E. J. Inorg. Biochem., 1994, 54, 167.

[16] Li, L.; Yang, J.; Wu, X.; Sun, Ch.; Zhou, G. J. Lumin., 2003, 101, 141.

[17] Yevdokimov, Y.M.; Salyanov, V.I.; Kondrashina, O.V.; Borshevsky, V.I.; Semenov, S.V.; Gasanov, A.A.; Reshetov, IV, Kuznetsov, V.D.; Nikiforov, V.N.; Akulinichev, S.V.; Mordovskoi, M.V.; Potashev, S.I.; Skorkin, V.M. Int. J. Biol. Macromol., 2005, 37, 165.

[18] Salayanov, V. I.; Evseev, A. I.; Popenko, V. I.; Gasanov, A. A.; Dembo, K. A.; Kondrashina, O. V. Biofizika, 2007, 52, 452

[19] Belyakov, V.A.; Orlov, V.P.; Semenov, S.V.; Skuridin, S.G.; Yevdokimov, Yu. M. Liq. Cryst., 1996, 20, 777.

[20] Gottarelli, G.; Spada, G.P.; In:”Circular Dichroism. Principles and Applications". Nakanishi, K.; Beroua, N.; Woody, R.W. (Eds.), VCH, N-Y., 1994.

[21] Yevdokimov, Yu. M.; Skuridin, S.G.; Semenov, S.V.; Salyanov, V.I.; Lortkipanidze, G.B. Biofizika, 1998, 43, 240.

[22] Keller, D.; Bustamante, C. J. Chem. Phys., 1985, 84, 2972.

[23] Kim, M.; Ulibarri, L.; Keller, D.; Maestre, M.F.; Bustamante, C. J. Chem. Phys., 1986, 84, 2981.

[24] Bouligand, Y. Am. Chem. Soc. Polym. Prepr., 1977, 18, 33.

[25] Yevdokimov, Yu. M.; Skuridin, S.G.; Nechipurenko, Yu. D.; Zakharov, M.A.; Salyanov, V.I.; Kurnosov, A.A.; Kuznetsov, V.D.; Nikiforov, V.N. Int. J. Biol. Macromol., 2005, 1-2, 103.

[26] Grasso, D.; Fasone, S.; La Rosa, C.; Salyanov, V. Liq. Cryst., 1991, 9, 299.

[27] Grasso, D.; Gabriele-Campisi, R. Liq. Cryst., 1993, 15, 701.

[28] Yu, M. Yevdokimov, Liq. Cryst. Pract. Appl., 2003, 3, 10 (in Russian).

[29] Livolant, F. Physica A, 1991, 176, 117.

[30] Durand, D.; Doucet, J.; Livolant, F. J. Phys. (France), 1992, 2, 1769.

[31] Livolant, F.; Leforestier, A. Prog. Polym. Sci., 1996, 21, 1115.

[32] Ha, S.C.; Lowenhaupt, K.; Rich, A.; Kim, Y-G.; Kim, K.K. Nature, 2005, $437,1183$.

[33] Lessing, P. A.; Erickson, A. W. J. Eur. Ceram. Soc., 2003, 23, 3049.

[34] Qi, Yu.-H.; Zvang, Q-Y.; Xu, L. J. Chem. Inf. Sci., 2002, 42, 1471.

[35] Bustamante, K.; Maestre, M. F.; Tinoco, I. J. Chem. Phys., 1980, 73, 6046 .

[36] Kim, Y. H. J. Physique, 1982, 43, 559.

[37] De Gennes, P.G. The Physics of Liquid Crystals, Clarendon Press, Oxford, 1974.

[38] Besteman, K.; van Eijk, K.; Lemay, S.G. Nat. Phys., 2007, 3, 641. 
[39] Rudd, L.; Lee, D.J.; Kornyshev, A. J. Phys. Condens. Matter, 2007, $19,416103$.

[40] Kornyshev, A. A.; Leikin, S.; Malinin, S.V. Eur. Phys. J., 2002, E7, 83.

[41] Grosberg, A. Yu.; Nguyen, T.T.; Shklovskii, B.I. Rev. Modern Phys., 2002, 74, 329.

[42] Osipov, M. A. Il Nuovo Cimento, 1997, 10D, 1249.

[43] Podgornik, R.; Parsegian, V. A. Phys. Rev. Lett., 1998, 80, 1560.
[44] Harris, A. B.; Kamien, R. D.; Lubensky, T. C. Rev. Mod. Phys., 1999, 71, 1745.

[45] Kornyshev, A. A.; Leikin, S. Phys. Rev., 2000, E 62, 2576.

[46] Gelbart, W. M.; Bruinsma, R. F.; Pincus, P. A.; Parsegian, V. A. Phys. Today, 2000, 53, 38

[47] Golo, V. L.; Kats, E. I.; Kikot', I. P. J. Exp. Theor. Phys. Lett., 2006, 84, 275.

(C) Yevdokimov et al.; Licensee Bentham Open.

This is an open access article distributed under the terms of the Creative Commons Attribution License (http://creativecommons.org/licenses/by/2.5/), which permits unrestrictive use, distribution, and reproduction in any medium, provided the original work is properly cited. 